\title{
Helmet Continuous Positive Airway Pressure in the Treatment of COVID-19 Patients with Acute Respiratory Failure could be an Effective Strategy: A Feasibility Study
}

\author{
Abdulrahman Alharthy ${ }^{1}$, Fahad Faqihi ${ }^{1}$, AlFateh Noor ${ }^{1}$, Ibrahim Soliman ${ }^{1}$, Peter G. Brindley ${ }^{2}$, \\ Dimitrios Karakitsos ${ }^{1,3, *}$,(D), Ziad A. Memish ${ }^{4,5,6, \text { (D) }}$ \\ ${ }^{1}$ Critical Care Department, King Saud Medical City, Riyadh, Kingdom of Saudi Arabia \\ ${ }^{2}$ Department of Critical Care, Faculty of Medicine and Dentistry, the University of Alberta, Alberta, Canada \\ ${ }^{3}$ Critical Care Department, Keck Medical School, University of Southern California, Los Angeles, CA, USA \\ ${ }^{4}$ Senior Infectious Diseases Consultant \& Director Research \& Innovation Center, King Saud Medical City, Ministry of Health, Riyadh, Saudi Arabia \\ ${ }^{5}$ Faculty of Medicine, Alfaisal University, Riyadh, Saudi Arabia \\ ${ }^{6}$ Hubert Department of Global Health, Rollins School of Public Health, Emory University, Atlanta, GA, USA
}

\section{ARTICLE INFO \\ Article History \\ Received 01 July 2020 \\ Accepted 13 August 2020 \\ Keywords \\ Helmet continuous positive airway pressure \\ high flow nasal cannula acute respiratory failure COVID-19}

(C) 2020 The Authors. Published by Atlantis Press International B.V. This is an open access article distributed under the CC BY-NC 4.0 license (http://creativecommons.org/licenses/by-nc/4.0/).

\section{INTRODUCTION}

COVID-19 pandemic caused by severe acute respiratory syndrome coronavirus-2 (SARS-COV-2) pandemic began in December 2019 and is still evolving [1]. Fortunately, most individuals are asymptomatic or have minor symptoms. However, a minority develop life-threatening disease: defined by Acute Respiratory Failure/Acute Respiratory Distress Syndrome (ARF/ARDS), septic shock, and Multi-system Organ Failure (MSOF) [2]. Along with uncertainty as to the role of pharmaceuticals [3], there is ongoing debate about how best to oxygenate patients. High Flow Nasal Cannula (HFNC) has shown promise for critically ill COVID-19 patients [4]. Continuous Positive Airway Pressure via a Helmet (H-CPAP) has been suggested, but there is limited published data [5]. We present feasibility data from 30 patients with COVID-19 and ARF who received H-CPAP $(n=15)$ and HFNC $(n=15)$ in our Intensive Care Unit (ICU).

The ICU of King Saud Medical City (Riyadh, Saudi Arabia) is a 200-bed polyvalent unit designated for COVID-19 by the Saudi Ministry of Health (MOH). As of April 30th, 2020, 380 patients with COVID-19 had been admitted to our ICU, including 320 critically ill patients, and 100 with moderate to serious disease. COVID-19

"Corresponding author.Email: karakitsosdimitrios@gmail.com was confirmed in all 380 patients by real-time-polymerase-chainreaction assays from nasopharyngeal swabs. Of 60 non-intubated patients on supplemental oxygen, 30 required higher support. Fifteen of these 30 received HFNC and 15 received H-CPAP. All 30 patients had serious COVID-19 pneumonia defined by ARF: dyspnea, respiratory rate $\geq 30 / \mathrm{min}$, blood oxygen saturation $\leq 93 \%$, ratio of partial arterial pressure of oxygen to fractional inspired concentration of oxygen $\left(\mathrm{PaO}_{2} / \mathrm{FiO}_{2}\right)<300$ and/or development of bilateral pulmonary infiltrates within 24-48 hours [6].

We retrospectively compared clinical data from the 30 patients by the two types oxygenation methods. Continuous variables are expressed as medians with interquartile range and categorical variables as absolute numbers or proportions. We utilized the Wilcoxon signed rank test for non-parametric data to compare the two groups, with $p$-value $<0.05$ considered significant. Statistical analysis was performed using SPSS, version 23.0.

The main characteristics of our 30 COVID-19 patients (15 HFNC; 15 H-CPAP) are outlined in Table 1. Major comorbidities observed were: hypertension (46.6\% in each group), diabetes mellitus (46.6\% HFNC vs. 33.3\% H-CPAP) and end-stage kidney disease on maintenance dialysis (6.6\% in each group). ICU supportive care was applied equally as per the Saudi $\mathrm{MOH}$ treatment protocol [6], as well as empiric therapy with lopinavir/ritonavir, ribavirin and 
Table 1 Characteristics of the COVID-19 patients who were treated with high flow nasal cannula $(n=15)$ and continuous positive airway pressure by means of a helmet $(n=15)$

\begin{tabular}{lcc}
\hline Characteristics & H-CPAP $(\boldsymbol{n}=\mathbf{1 5})$ & HFNC $(\boldsymbol{n}=\mathbf{1 5})$ \\
\hline Age (years) & $46(38-55)$ & $44(37-57)$ \\
Sex (male/female) & $12 / 3$ & $13 / 2$ \\
Body mass index $\left(\mathrm{kg} / \mathrm{m}^{2}\right)$ & $24(20-29)$ & $24(20-29)$ \\
Sequential Organ Function & $9(8-10)$ & $9(8-10)$ \\
$\quad$ Assessment score & $4(3-5)$ & $4(3-5)$ \\
Pneumonia severity index & $8(6-11)$ & $9(7-11)$ \\
Treatment time (days) & 40 & $100^{+}$ \\
Awake prone positioning $(\%)$ & $7.5(5.2-10.4)$ & $7.6(5.3-10.6)$ \\
ROX index after 2 h treatment & $8.2(6.4-11.1)$ & $8.4(6.7-11.2)$ \\
$\mathrm{ROX}_{\text {index after 12 h treatment }}$ & $211(198-235)$ & $213(199-241)$ \\
$\mathrm{PaO} / \mathrm{FiO}_{2}$ ratio before treatment & $377(344-422)$ & $380(352-421)$ \\
$\mathrm{PaO}_{2} / \mathrm{FiO}_{2}$ ratio after treatment & 20 & 13.3 \\
$\mathrm{Intubation} \mathrm{rate}(\%)$ & &
\end{tabular}

" $p<0.05$. Values are medians with interquartile ranges. ROX index, rate of oxygenation index.

interferon beta-1b for 14-days, dexamethasone for 10 days, and prophylactic anticoagulation. Upon ICU admission, chest imaging showed bilateral peripheral ground-glass opacities and variable consolidations in all 30 patients, mainly in the posterior and lower lung fields. All patients were severely hypoxemic and required high level oxygen supplementation. The $\mathrm{PaO}_{2} / \mathrm{FiO}_{2}$ ratio, Sequential Organ Function score, and pneumonia severity index were similar between the two groups (Table 1).

The Rate of Oxygenation Index (ROX = oxygen saturation/(fraction of inspired oxygen $\times$ respiratory rate) was used to assess the effect of applied oxygen therapies after 2 and $12 \mathrm{~h}$ as described elsewhere [7]. Both groups showed full treatment compliance. ROX remained greater than five throughout in all patients, indicating successful oxygenation. Notably, these oxygen therapies were complemented by awake prone positioning (12-20 h/day) in all HFNC patients but only $40 \%$ of H-CPAP patients, due to the helmet's volume and circuit related issues $(p<0.05)$. During the first day of application, HFNC was adjusted at a median flow rate of $60 \mathrm{~L} / \mathrm{min}$ and median fraction of inspired oxygen of $40 \%$; while the helmet was adjusted to deliver CPAP at high flow rates to prevent rebreathing (median flow rate $45 \mathrm{~L} / \mathrm{min}$ ) with a median fraction of inspired oxygen of $40 \%$. The duration of treatment and the intubation rates were comparable between the two groups (Table 1).

Our preliminary data suggests that HFNC is feasible in patients with ARF from COVID-19 pneumonia, and effective in maintaining oxygenation. H-CPAP is similarly effective in terms of oxygenation but could not be maintained in over half-of-all patients when proned. However, there are putative advantages to H-CPAP. Importantly, H-CPAP includes a soft silicon collar which forms a pneumatic seal around the patient's neck, and contains an expiratory filter: both of which should decrease viral spread. A heat-moisture exchange filter on the gas inspiratory limb reduces the noise level inside the helmet which should increase tolerance. Both oxygen delivery systems deliver humidified gas to the upper airway and therefore should reduce the metabolic work related to gas conditioning [4]. H-CPAP also incorporates a distal variable CPAP valve and ports for the insertion of feeding tubes or the administration of nebulizers (Figure 1) [5,8].

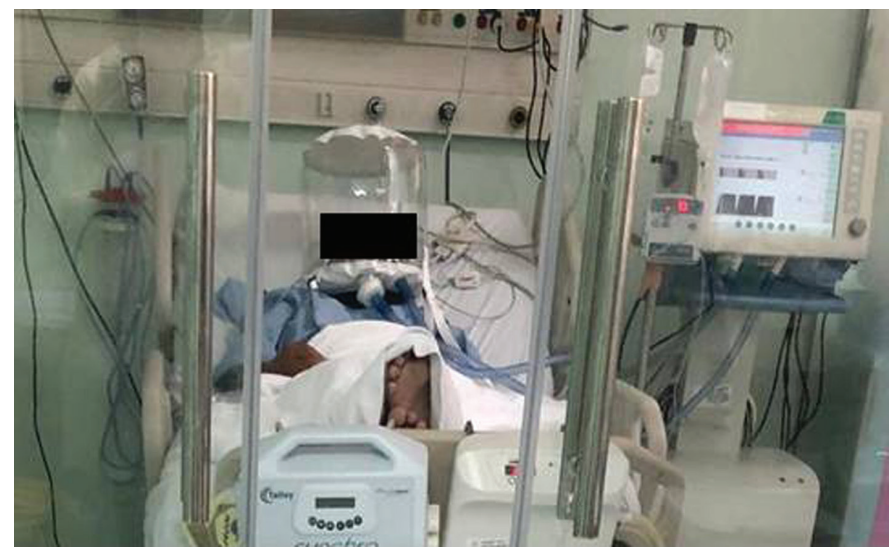

Figure $1 \mid$ One of our COVID-19 patients (face is covered to ensure anonymity) receiving continuous positive airway pressure by means of a helmet. Please, observe the patient's level of comfort and the helmet's connection to the conventional ventilator.

This pilot study has limitations which preclude its generalizability. First the number of patients was small thus prevented meaningful statistical analysis. Oxygen was also delivered in a controlled ICU environment, not a general ward, or a (potentially chaotic) emergency department. Following this feasibility study we also need to determine comparative aerosol generation with HFNC and H-CPAP, given that this concern has substantially deterred their usage. Importantly, we are also not asserting that intubation can (or should) be avoided in COVID-19 patients using HFNC or H-CPAP. Contrasting anecdotal reports have suggested that these methods can prevent intubation, while others counter that it merely delays inevitable intubation while reducing patient's cardiopulmonary reserve. Clearly, larger randomized control trials are required to confirm or refute these hypotheses, and to define the optimal role for HFNC and H-CPAP in COVID-19.

\section{CONFLICTS OF INTEREST}

The authors declare they have no conflicts of interest.

\section{AUTHORS' CONTRIBUTION}

AA, PGF, ZAM and DK have designed this study and drafted the manuscript. FF, AN and IS collected the data and performed the analysis. All authors have equally drafted and reviewed the final version of this manuscript.

\section{FUNDING}

No financial support was provided.

\section{ETHICAL APPROVAL}

The study was approved by our Institutional Review Board. Patients or their legal representatives provided a written consent for the use of their imaging and clinical data for scientific purposes. 


\section{REFERENCES}

[1] Guan WJ, Ni ZY, Hu Y, Liang WH, Ou CQ, He JX, et al. Clinical characteristics of coronavirus disease 2019 in China. N Engl J Med 2020;382;1708-20.

[2] Zhou F, Yu T, Du R, Fan G, Liu Y, Liu Z, et al. Clinical course and risk factors for mortality of adult inpatients with COVID-19 in Wuhan, China: a retrospective cohort study. Lancet 2020;395;1054-62.

[3] Grein J, Ohmagari N, Shin D, Diaz G, Asperges E, Castagna A, et al. Compassionate use of remdesivir for patients with severe Covid-19. N Engl J Med 2020;382;2327-36.

[4] Geng S, Mei Q, Zhu C, Yang T, Yang Y, Fang X, et al. High flow nasal cannula is a good treatment option for COVID-19. Heart Lung 2020;S0147-9563(20)30113-8.
[5] Ing RJ, Bills C, Merritt G, Ragusa R, Bremner RM, Bellia F. Role of helmet-delivered noninvasive pressure support ventilation in COVID-19 patients. J Cardiothorac Vasc Anesth 2020;S10530770(20)30419-5.

[6] Saudi Ministry of Health. Coronavirus Diseases 19 (COVID-19) guidelines (revised version 1.7). 2020. Available from: https:// covid19.moh.gov.sa.

[7] Roca O, Caralt B, Messika J, Samper M, Sztrymf B, Hernández $\mathrm{G}$, et al. An index combining respiratory rate and oxygenation to predict outcome of nasal high-flow therapy. Am J Respir Care Med 2019;199;1368-76.

[8] Lucchini A, Giani M, Isgrò S, Rona R, Foti G. The "helmet bundle" in COVID-19 patients undergoing non invasive ventilation. Intensive Crit Care Nurs 2020;58;102859. 